\title{
Aproximación a la realidad aumentada y virtual como herramienta didáctica pedagógica: Tecnología con un enfoque a las etnociencias
}

\author{
Daysi Pamela Castellanos Quiroga \\ Universidad Central del Ecuador \\ dpcastellanos@uce.edu.ec \\ Mauricio Xavier Melo Castro \\ Universidad Central del Ecuador \\ mxmelo@uce.edu.ec
}

Recibido: 9 - febrero - 2018 / Aceptado: 31 - febrero - 2018

\section{Resumen}

La tecnología como la educación deben avanzar a la par, por lo tanto, es necesario adaptar estos conceptos al proceso de enseńanza de los estudiantes de las futuras generaciones, pero sin perder el concepto de lo que en realidad es la educación y su uso adecuado a través de las tecnologías. Existe una nueva tendencia en tecnología educativa que engloba dos instancias, conocidas como Realidad Aumentada y Realidad Virtual. La recopilación de experimentos en distintas instituciones importantes, donde se demuestra el éxito de esta tendencia en el campo de la educación y su potencial integración con las Etnociencias, sin embargo son poco conocidas y utilizadas por los docentes en la actualidad, sobre todo en nuestro medio, por lo tanto es necesario que se conozca su utilización y beneficios, además de las aplicaciones educativas, generando una cultura que utilice estas herramientas para el beneficio de la sociedad. 
Palabras clave: educación, tecnología, realidad virtual, realidad aumentada, etnociencias.

\section{Abstract}

Technology and education must advance at the same time, therefore, it is necessary to adapt these concepts to the teaching process of students of future generations, but without losing the concept of what education really is and its proper use through technologies. There is a new trend in educational technology that encompasses two instances, known as Augmented Reality and Virtual Reality, The recompilation of experiments in different important institutions demonstrates the success of this trend in the field of education and its potential integration with ethnosciences, however are little known and used by teachers today, especially in our environment, therefore it is necessary to know its use and benefits, in addition to educational applications, generating a culture that uses these tools for the benefit of society.

Keywords: education, technology, virtual reality, augmented reality, ethnosciences. 


\section{¿Qué entendemos por tecnología con un enfoque didáctico y por qué es importan- te en el contexto de las Etnociencias?}

$\mathrm{E}$ sta pregunta abre dos enfoques, el campo de la tecnología aplicado al campo de la educación y su integración con las Etnociencias. El punto de partida tomado para dicho enfoque comienza desde la didáctica y su interacción en los procesos educativos los cuales según González (2016), constan de un ciclo compuesto por varios subprocesos, teniendo en consideración que desde un inicio se componen de un propósito y un interés los cuales corresponden a las habilidades que el docente quiere que se desarrollen a través de dicha herramienta didáctica. A su vez la didáctica es llamada una ciencia tecnológica por Gallego (2011) en la que expone que es una ciencia aplicada, práctica, y significa saber hacer.

Al referirnos al campo tecnológico tenemos que delimitar con qué instrumentos podemos trabajar para conseguir el enfoque deseado, en este caso utilizamos las "Nuevas Tecnologías" las cuales pueden ser utilizadas como instrumentos didácticos para facilitar y mejorar el proceso de enseñanza. El término TIC es la abreviación de Tecnologías de la Información y la Comunicación, que hace referencia al conjunto de avances tecnológicos que tenemos a nuestra disposición, pero no tiene el enfoque educativo que se busca dentro de la didáctica, para esto utilizaremos otro término como explica Ocańa (2013); las TAC por su abreviación Tecnologías del Aprendizaje y el Conocimiento para referimos a la adecuada aplicación de las TIC en un entorno educativo.

Para enfatizar la última línea temática, las Etnociencia son consideradas un conjunto de saberes ancestrales y científicos tomados en cuenta desde tres perspectivas fundamentales: antropológicas, históricas y educativas, que respondan a las necesidades reales de la sociedad ecuatoriana y andina, proponiendo una cosmovisión que integre las miradas prehispánicas y las epistemes occidentales.

\section{Por qué utilizar nuevas tecnologías}

Los estudiantes de las nuevas generaciones necesitan nuevas formas de enseñanza a las que comúnmente estamos acostumbrados, como lo expone Daura (2016, pág. 9) "Desde el nacimiento están en contacto con las nuevas tecnologías. Para ellos la computadora, los teléfonos móviles, forman parte de una realidad natural, equiparable a lo que para las generaciones anteriores son el libro, la agenda de papel y el reloj pulsera". Por lo que una correcta utilización de los recursos tecnológicos favorece plenamente al proceso de enseńanza que necesitan los estudiantes en la actualidad y de esta manera modificar la forma de obtener conocimiento significativo. Además, no solo favorece a los estudiantes de las nuevas generaciones ya que su utilización es de gran ayuda tanto para personas afines a la tecnología como para aquellos que no interactúan de manera constante con ella.

\section{¿Qué es la Realidad Aumentada y la Realidad Virtual?}

El primer término es conocido como Realidad Aumentada o "AR", es una tecnología que integra o combina la realidad física con datos los cuales son generados 
por ordenadores, (Galán, 2014, pág. 3) manifiesta que la realidad aumentada "Se encarga de introducir información virtual a nuestro entorno real". En este caso la tecnología a utilizar será basada en el uso de los dispositivos electrónicos y digitales que manejamos a diario como: teléfonos celulares inteligentes o "Smartphones", tabletas, computadores e internet, además existen aplicaciones especializadas para potenciar estos dispositivos.

El segundo término y en el que haremos un mayor énfasis es la Realidad Virtual o "VR" la cual es una tecnología que se encuentra en auge y corresponde a la interacción del ser humano para visualizar o experimentar un lugar físico o ficticio a través de un computador simulando un ambiente, como lo explica (Porras, 2000, pág. 13), "la Realidad Virtual a diferencia de una simple fotografía, hace que el usuario interactúe en un medio ambiente más aceptable para el proceso cognitivo. La representación de la computadora debiera ser por lo tanto más cercana a la realidad, si es que el mundo lo pretende".

\section{Realidad Aumentada como herramienta didáctica}

La Realidad Aumentada está teniendo un impacto positivo en la educación ya que es considerada por muchos el futuro de la enseñanza, como lo explica Muñoz (2017) quien propone que es fundamental incluir la realidad aumentada dentro de la educación ya que nos ayudará a hacer más dinámica y motivadora la práctica docente. $\mathrm{Si}$ bien en la etapa universitaria no es considerado tan didáctico, a nivel de primaria y secundaria es una herramienta fundamental para experimentar visual y auditivamente.

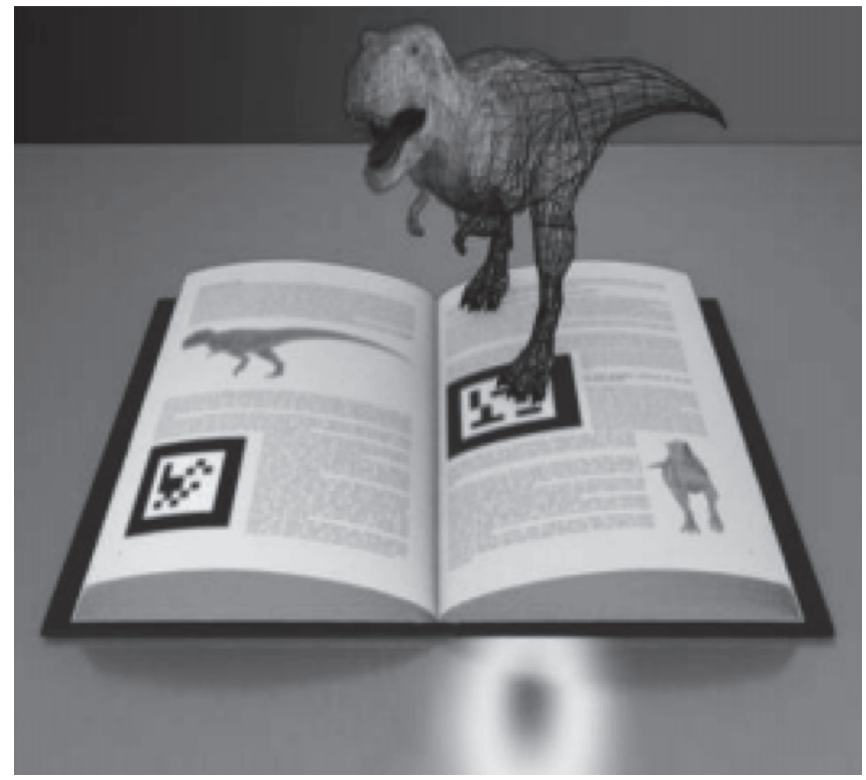

Figura 1. Realidad aumentada en libros de texto. Recuperado de https://biblioteca.ucm.es 


\section{Realidad Virtual como herramienta didáctica}

La Realidad Virtual o "VR" es una herramienta que crea un ambiente simulado, esta herramienta es muy útil en la educación ya que permite viajar y conocer procesos sin la necesidad de salir del salón de clase; se la puede utilizar en diferentes ámbitos de la educación porque contiene gran cantidad de contenidos, pero centrándose en el objetivo de desarrollar una clase para crear conocimientos en los estudiantes. El aprendizaje mediante la Realidad Virtual se centra en cumplir funciones de simulación mediante la cual se crea un aprendizaje simbólico. Además de mejorar las funciones cognitivas, el objetivo de esta herramienta tecnológica es la comprensión de contenidos, aparte que es mucho más económica que comprar materiales físicos para cada estudiante.

Un claro ejemplo es la aplicación de Celestron, SkyPortal la cual permite ver constelaciones en la pantalla del celular mediante un cielo simulado al igual que un planetario.

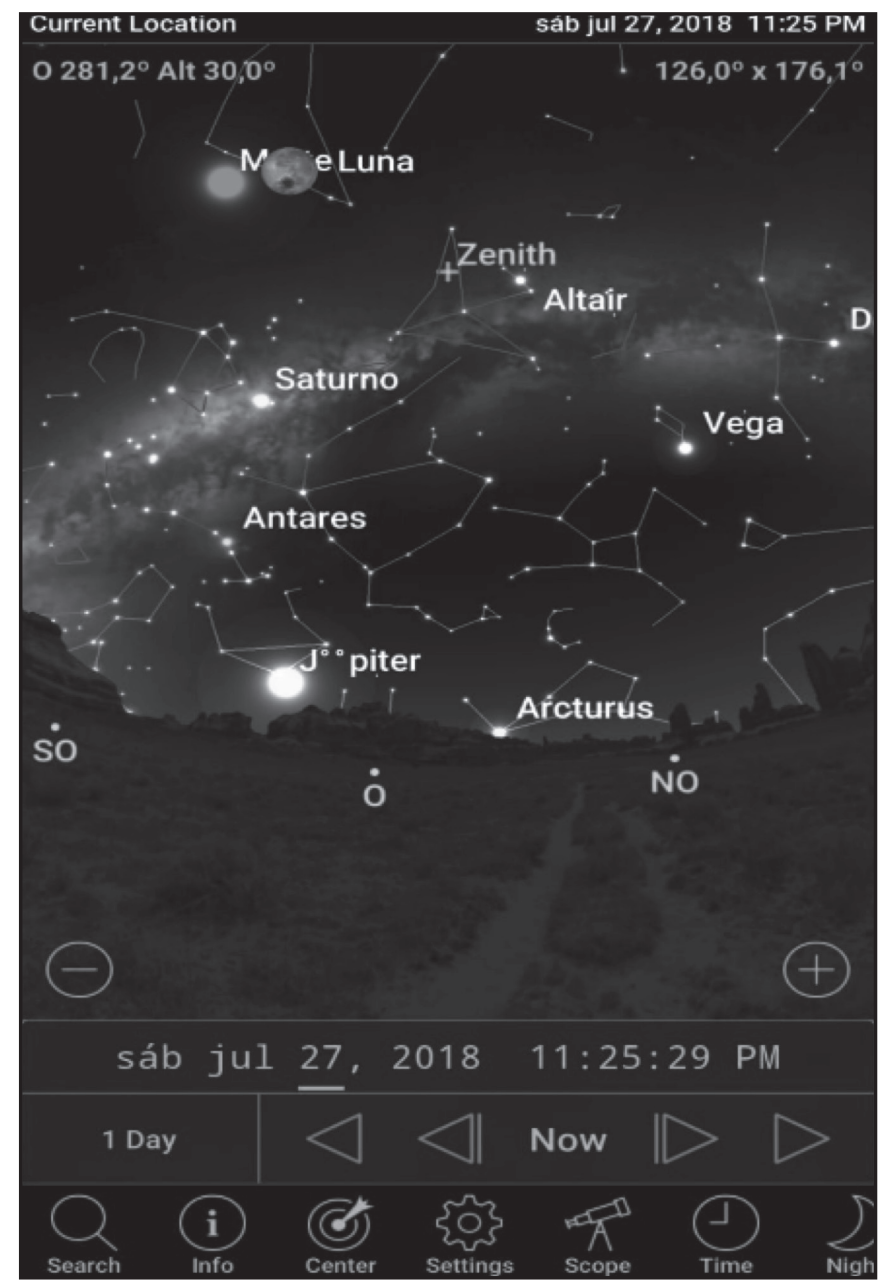

Figura 2. Realidad virtual.

Tomada desde aplicación: SkyPortal 


\section{Realidad Aumentada y Realidad Virtual aplicadas para la enseñanza de Etnociencias}

La Realidad Aumentada y Virtual no son un sustituto de un profesor, más bien se convierten en un apoyo en cuanto a las explicaciones o confusiones que puedan surgir en los estudiantes, además involucra al estudiante con la situación a manera de inmersión y la respalda con información en los dispositivos móviles, el aprender se hace notable por medio de resolución de problemas que pueden ocurrir en el ámbito real por lo que integra los aspectos mencionados anteriormente ya que es fundamental para entender a las Etnociencias.

Estas herramientas fueron aplicadas por el instituto Hunters Lane donde se ocuparon equipos donados por AMD y HTC Vive. 1700 estudiantes utilizaron los equipos para recibir clases de acuerdo con los temas planteados en los currículos, todos los alumnos podían observar lo que sucedía en los espacios virtuales permitiendo a los profesores hacer la clase más práctica. Según explica Ashley Ross, profesor de Tecnologías de la Información en Hunters Lane "el uso de la realidad virtual ha ayudado a los estudiantes a retener los conocimientos de forma más eficiente. Creo que esta tecnología podría cambiar las reglas del juego para los chicos que tienen más dificultades de aprendizaje ya que les sumerge en un mundo en el que pueden ver y sentir lo que deben aprender. Eso hace que las clases sean más interesantes y les 'engancha' mucho más" ( Ross, 2018, pág. 2).

En la Universidad de Stanford se trabaja con aplicaciones en escuelas para tratar habilidades emocionales en los niños. "Emoti" es un ejercicio de atención hecho para realidad virtual, creada por Facebook y el Centro de Inteligencia Emocional de Yale, la cual permite experimentar juegos de rol y ejercicios de confianza para mejorar las habilidades sociales en los estudiantes.

El que caso concreto de la utilización para la enseñanza de Etnociencias consiste, por ejemplo, en el diseño de ambientes donde el estudiante pueda identificar la problemática social y el contexto científico-histórico como lo demuestra la aplicación Google-Expeditions creada por la compañía Google la cual como explica en su página principal "Los profesores pueden usar dispositivos móviles para llevar objetos virtuales a su clase para que los alumnos, utilizando teléfonos móviles, puedan ver y caminar virtualmente alrededor de los objetos $3 \mathrm{D}$ como si estuvieran físicamente en el aula". (cita de la web)

Con la ayuda de la aplicación ARCore, Tim Brzezinski Consultor independiente de educación matemática en la Universidad Estatal de Connecticut llegó a la modelación a través del software GeoGebra de un cuenco con características prehispánicas proyectado con RA. 


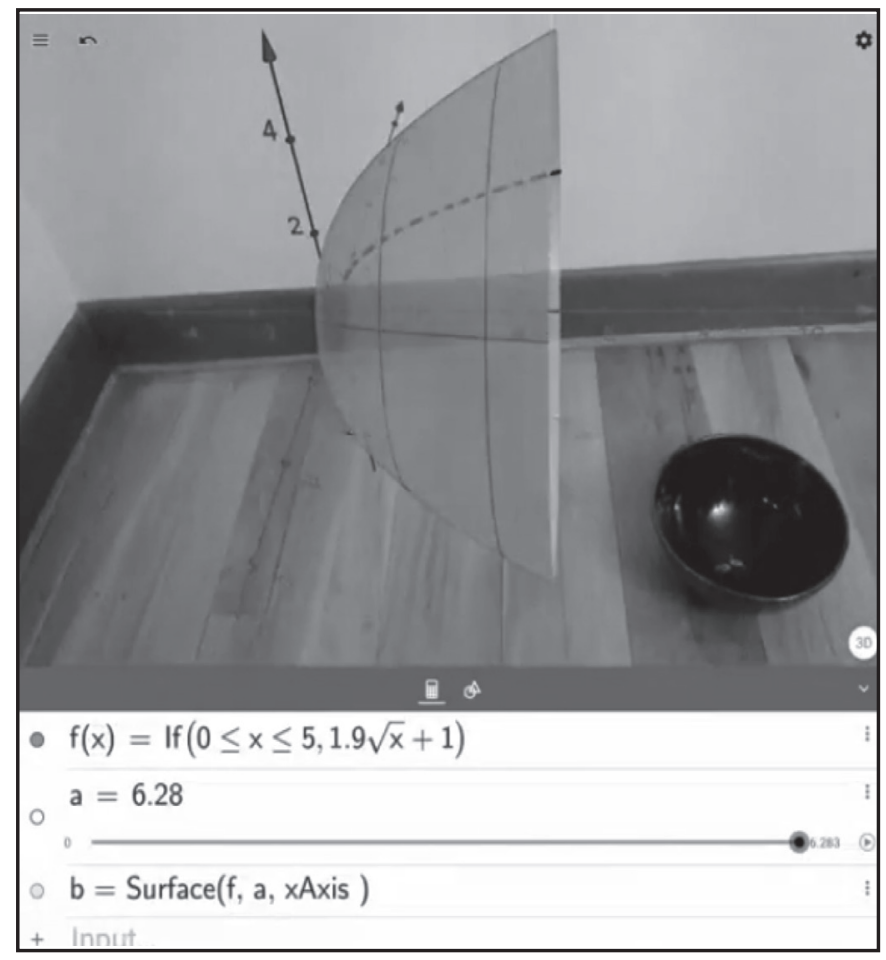

Figura 3. Realidad aumentada modelación de un cuenco. Recuperado de: https://www.youtube.com/watch?v=UMFsM1_YmOA

\section{Conclusión}

La Realidad Virtual y Aumentada debe ser llevada al campo de la educación en el Ecuador ya que se ha demostrado su influencia dentro del proceso de enseñanza-aprendizaje. Esta se presenta como una alternativa al marco de educación tradicional en el cual estudiantes interactúan directamente con el contenido.

La educación interactiva y en el caso particular integrada a las Etnociencias satisface las necesidades educativas de las generaciones actuales y futuras además de potenciar un aprendizaje más rápido y efectivo, por esta razón la evolución de la tecnología y la educación deben ir de la mano para formar seres con capacidades de resolución de problemas de una manera crítica, constructiva y forjadora de investigación formativa.

\section{Referencias}

Daura, F. T.-B. (2016). El desafío de enseñar a la generación Net. En Aprender a enseñar. Buenos Aires: falta la editorial. 
Galán, L. (2014). Realidad aumentada. Interacción persona-ordenador. Ciudad: editorial.

Google. (09 de 10 de 2018). Google-Expeditions. Obtenido de Https://edu.google.com/

Gallego, M. R. (2011). Didáctica y currículum para el desarrollo profesional. Ciudad: editorial.

González, G. E. (2016). Procesos pedagógicos y didácticos de una sesión de aprendizaje. Lima: editorial

Medina, A. y Mata, F. (2002). Didáctica general. Madrid: Pearson.

Ocaña, M. C. (2013). Integración de las TACS en educación. Valencia: editorial

Porras, P. A. (2000). Realidad virtual. Puebla: editorial

Muñoz, C. M. (2017). "Aplicaciones de la realidad aumentada”. En La educación. Madrid: E-Innova Bucm. 